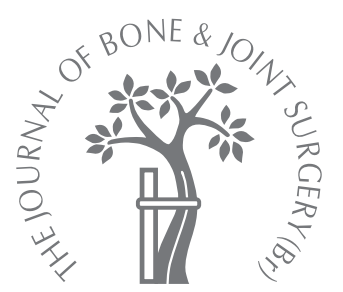

\title{
Impaction grafting with a bone-graft substitute in a sheep model of revision hip replacement
}

\author{
M. Coathup, \\ N. Smith, \\ C. Kingsley, \\ T. Buckland, \\ R. Dattani, \\ P. Ascroft, \\ G. Blunn
}

From University

College, London,

England

\begin{abstract}
An experimental sheep model was used for impaction allografting of $\mathbf{1 2}$ hemiarthroplasty femoral components placed into two equal-sized groups. In group 1, a 50:50 mixture of ApaPore hydroxyapatite bone-graft substitute and allograft was used. In group 2, ApaPore and allograft were mixed in a 90:10 ratio. Both groups were killed at six months. Ground reaction force results demonstrated no significant differences $(p>0.05)$ between the two groups at 8, 16 and 24 weeks post-operatively, and all animals remained active. The mean bone turnover rates were significantly greater in group 1, at $0.00206 \mathrm{~mm} /$ day, compared to group 2 at $0.0013 \mathrm{~mm} /$ day $(p<0.05)$. The results for the area of new bone formation demonstrated no significant differences $(p>0.05)$ between the two groups. No significant differences were found between the two groups in thickness of the cement mantle $(p>0.05)$ and percentage ApaPore-bone contact $(p>0.05)$.

The results of this animal study demonstrated that a mixture of ApaPore allograft in a 90:10 ratio was comparable to using a 50:50 mixture.
\end{abstract}

The increasing practice of undertaking total hip replacement (THR) in younger patients has been associated with a doubling of the rates of revision THR since 1991, with one in five of all THRs now being a revision procedure. $^{1,2}$ Patients with bone loss may face multiple revisions as a result of inadequate fixation of the stem, reducing the survival of revision prostheses and exacerbating bone loss. ${ }^{3}$ Impaction allografting has the potential to regenerate proximal bone and increase fixation of the implant in the proximal femur. Studies have shown encouraging clinical, radiological and histological results, demonstrating that impaction bone grafting can reverse loss of bone stock, with graft being replaced by new bone in both humans and in animal models. ${ }^{4}$ However, allograft does have a number of disadvantages; it can evoke an immune response, it lacks osteogenic cells, which are destroyed by freezing, and it increases the risk of infection. ${ }^{5}$ The material properties of bone graft obtained from different femoral heads are inconsistent. Recent research has centred on maximising osteoconduction and the capacity for bone incorporation of impaction grafting when using bone-graft substitutes such as hydroxyapatite (HA). ${ }^{6,7}$ Several commercial bone-graft substitutes are now used to expand the quantities of allograft available.
One such substitute is ApaPore 60, made from HA (ApaTech Ltd, Elstree, United Kingdom) which has been chemically and structurally manipulated to maximise host bone ingrowth following impaction while maintaining structural integrity. ApaPore 60 granules, which measure between $2 \mathrm{~mm}$ and $5 \mathrm{~mm}$, have a microporosity similar to that of cancellous bone, which allows nutrient transfer and enhances graft stability. In addition, it has a high level of fully interconnected macroporosity, which facilitates angiogenesis and osseointegration. The aim of this study was to test the hypothesis that the use of ApaPore in two combinations with allograft would be stable and induce similar patterns of bone formation in impaction grafting in the femur.

\section{Materials and Methods}

Femoral hemiarthroplasty components were inserted into the right hips of 12 skeletallymature female commercially cross-bred sheep weighing between $65 \mathrm{~kg}$ and $80 \mathrm{~kg}$ and aged between two and five years. All procedures were carried out in compliance with United Kingdom Home Office regulations (Animal Scientific Procedures Act 1986). ${ }^{8}$ The prostheses were manufactured specifically for a sheep model from cast cobalt-chrome alloy of a collarless, polished double-tapered design and were cemented in place. Two groups of six 


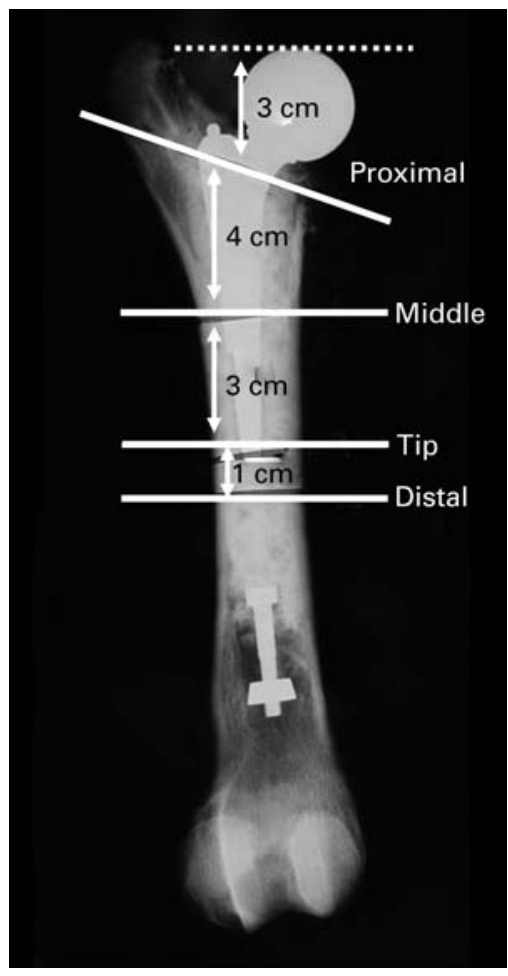

Fig. 1

An anteroposterior radiograph of a retrieved femur demonstrating the levels at which the implant was sectioned and analysed.

animals were used. In group 1, the ApaPore was mixed with allograft in a 50:50 ratio by volume, and in group 2 the ApaPore:allograft ratio was 90:10.

Graft preparation. Prior to surgery, allograft was harvested from the epiphyses of sheep femora and tibiae and morcellised to produce a graded graft material with a bone chip size of less than $5 \mathrm{~mm}$. Our graft was gamma irradiated in a frozen state with $25 \mathrm{kGy}$ to $35 \mathrm{kGy}$ and stored at $-20^{\circ} \mathrm{C}$ with thawing undertaken approximately two hours before surgery.

Surgery. The right hip joint was exposed through an anterolateral approach and the femoral head excised. The small amount of cancellous bone in the proximal femur was removed using a curette. A centralising guide wire was attached to an expandable restrictor which was placed in the cavity, and the distal graft was impacted in place using modified Exeter impactors (Stryker UK Ltd, Newbury, United Kingdom) which fitted over the guide wire. Prior to insertion, the ApaPore granules and allograft were mixed in blood (1:1 mixture) withdrawn from the jugular vein. Once coagulated, the mixture was inserted and impacted within the femur to a thickness of $3 \mathrm{~mm}$ to $4 \mathrm{~mm}$. The guide wire was removed, the cavity filled with surgical grade Simplex bone cement (Stryker UK Ltd) using a cement gun and a revision insert (Stryker UK Ltd), and the femoral compo- nent inserted after 3.5 to 4 minutes. In all cases, a $25 \mathrm{~mm}$ diameter femoral head was used. The hip joint was then reduced and the wound closed.

After surgery, animals were allowed immediate mobilisation and weight-bearing as tolerated. Antibiotic and analgesic prophylaxis was administered daily with subcutaneous injections of Baytril $(5 \mathrm{mg} / \mathrm{kg}$ enrofloxacin; Bayer AG, Leverkusen, Germany) and Finadyne (2 mg/45 kg flunixin meglumine; Schering-Plough Ltd, Uxbridge, United Kingdom) for three days post-operatively. Animals were kept in individual pens for four weeks before being housed together. Implants remained in vivo for six months. Ground reaction force. The animals were walked over a Kistler force plate (Kistler Instrument Corp., Amherst, New York) both before operation and at 8, 16 and 24 weeks after. A total of 12 readings of maximum force $\left(\mathrm{F}_{\max }, \mathrm{N} / \mathrm{m}^{2}\right)$ were taken for the right and left legs of each animal. Mean values of right over left were calculated as a percentage $(\% \mathrm{mR} / \mathrm{mL})$ to evaluate how well the animal used its operated leg, where $100 \%$ represented full weight-bearing. Preoperatively, only animals that were within a $5 \%$ range of full weight-bearing were selected for use in the study.

Radiological analysis. Radiographs of each hip joint were taken immediately following operation, at 8 and 16 weeks after, and then following killing after 24 weeks. These radiographs were used for observation only.

On retrieval, the femora were removed and immediately placed in a $10 \%$ formaldehyde solution and, following dehydration, embedded in hard-grade acrylic resin (LR White; London Resin Company, London, United Kingdom). Sections approximately $60 \mu \mathrm{m}$ thick were prepared through four regions of the femur comprising the proximal, middle and tip of the femoral component, and one region distal to the tip (Fig. 1) by cutting on a diamond band saw, followed by grinding using abrasive papers and finished by polishing on a cloth with aluminium oxide.

Tetracycline bone marking. Fluorescent bone markers were administered to allow quantification of the rate of bone turnover adjacent to the femoral component in the two experimental groups. Subcutaneous injections $(30 \mathrm{mg} / \mathrm{kg})$ were given two months post-operatively and three weeks later, followed by a third administration in the fifth month after surgery, and another three weeks later. Prior to staining, the $60 \mu \mathrm{m}$ sections were examined using fluorescence microscopy and photographed to record the fluorochrome lines. The rates of bone turnover in $\mu \mathrm{m} / \mathrm{day}$ were calculated by measuring the distance between these fluorescent lines, dividing by the magnification and the number of days between doses.

Histological analysis. Sections were prepared with toluidine blue and paragon staining. Images of each thin section were obtained using a digital camera and captured with the KS300 Image Analysis Software Package (Imaging Associates; Zeiss, Oxford, United Kingdom). Each section was divided into four quadrants, and using a power four objective lens, one random area within each quadrant within the 


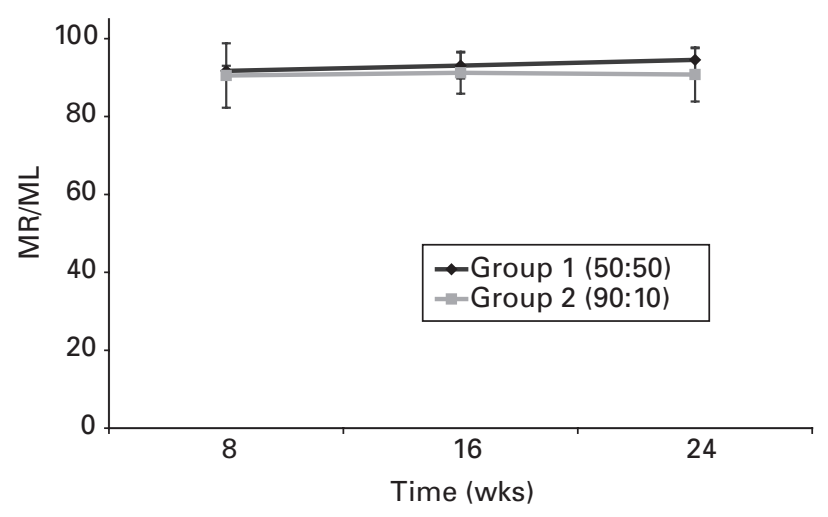

Fig. 2

A graph comparing the mean ground reaction force in the two experimental groups at three intervals post-operatively. Error bars show the standard errors.

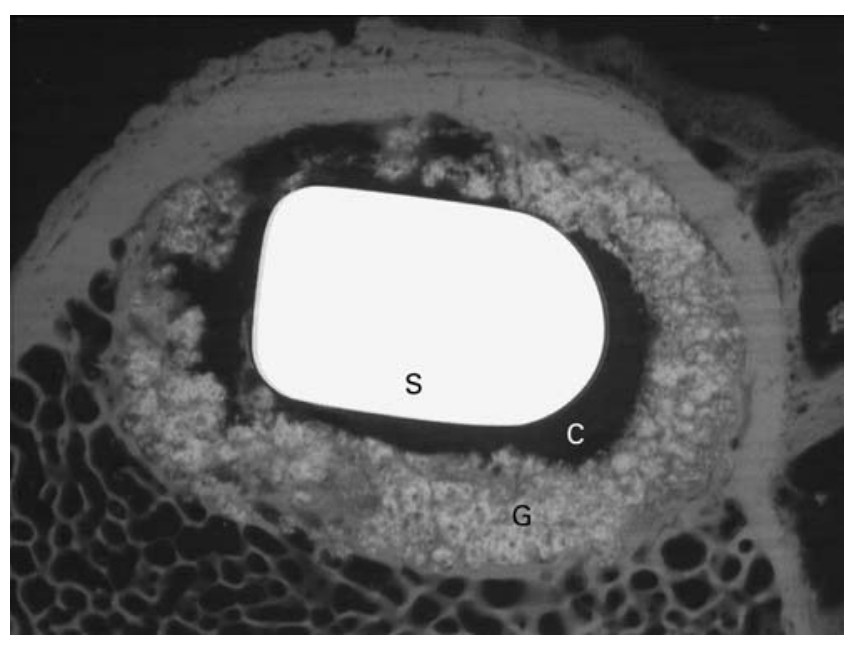

Fig. 3

A microradiograph of a transverse section through the proximal region of the femur of a sheep in group $2(90: 10)$ demonstrating impacted graft material (G) surrounding the implant stem (S) and bone cement (C).

borders of the original endosteal cavity was selected and the thickness of the cement mantle and the area of new bone formation was estimated using a threshold routine in the image analysis software package. The extent of contact between ApaPore and new bone was measured at eight equidistant sites using a line intercept method.

Scanning electron microscopy. Thin sections $(60 \mu \mathrm{m})$ were sputter-coated with gold and prepared for viewing using the Jeol Winesem JSM-35C scanning electron microscope (SEM) (JEOL, Welwyn Garden City, United Kingdom). Only the distal sections from each animal in each experimental group were assessed using this technique. Specimens were examined using backscattered electron microscopy.

Statistical analysis. The results were analysed using the SPSS version 10.1 statistical software package (SPSS Inc., Chicago, Illinois). A Kolmogorov-Smirnov test showed that all data were non-parametric, and a Mann-Whitney U test was used to compare results obtained in the different experimental groups. A p-value $<0.05$ was considered significant.

\section{Results}

Ground reaction force. No significant differences were observed in the mean pre-operative ground reaction force when the experimental groups were compared (MannWhitney $\mathrm{U}$ test, $\mathrm{p}>0.05)$. All results were normalised to the mean $\% \mathrm{mR} / \mathrm{mL}$ values collected pre-operatively (mean 100.82 (standard deviation (SD) 0.63)). No significant differences in the ground reaction force were identified when groups 1 and 2 were compared at 8, 16 and 24 weeks (Mann-Whitney U test, $\mathrm{p}>0.05$ ). No significant differences were demonstrated in animals in group 2 at any stage compared to pre-operative values. In contrast, animals in group 1 all demonstrated a significantly decreased ground reaction force at 8, 16 (mean 93.09 (SD 3.33); Mann-Whitney U test, $\mathrm{p}=0.021$ ) and 24 weeks (mean 95.44 (SD 2.99)); Mann-Whitney U test, $\mathrm{p}=0.035$ ) compared with pre-operative values. In both groups the ground reaction force increased steadily over time until retrieval (Fig. 2).

Radiography. Anteroposterior (AP) and mediolateral radiographs of the retrieved femora demonstrated all implants surrounded by densely impacted ApaPore-allograft composite. The implants appeared to be well fixed, with no obvious signs of subsidence. Slab radiographs through transverse slices ( $2 \mathrm{~mm}$ thick) of the harvested femora with the implant in situ showed that the cemented stem was surrounded by a thick layer of graft material (Fig. 3). Radiographs of transverse sections distal to the tip of the stem showed that the intramedullary cavity within the tubular cortical bone was packed with graft material.

Tetracycline labelling. Cement lines were evident separating newer areas of bone, and the markers were incorporated into both remodelled haversian systems in a regular concentric, centrifugal manner, and in areas of bone where layered appositional growth had occurred.

Qualitative observation demonstrated that in each of the groups bone turnover was greatest at the periphery at the endosteal surface of bone, and became less towards the cement surface. When the results for the fluorescent markers within the graft material from all four regions were presented as means, they demonstrated significantly greater bone turnover rates in group 1 at $2.060 \mu \mathrm{m} /$ day (SD 0.081) compared with group 2 at $1.580 \mu \mathrm{m} /$ day (SD 0.054) (MannWhitney U test, $\mathrm{p}>0.05)$. However, variations were found in bone turnover in the proximal, middle, tip and distal regions adjacent to the femoral component in the femur with the significantly highest bone turnover in the distal region in group $1\left(2.600 \mu \mathrm{m} / \mathrm{day}^{1}\right.$ (SD 0.014) compared 
with group $2(1.300 \mu \mathrm{m} /$ day (SD 0.05) (Mann-Whitney U test, $\mathrm{p}<0.05$ ) (Fig. 4a). No significant differences were observed in any of the other regions when the two groups were compared.

Thickness of the cement mantle. The results demonstrated no significant differences $(p>0.05)$ in the thickness of the cement mantle between the experimental groups, and when the proximal, middle and tip regions adjacent to the femoral stem were compared with a thickness of between $2 \mathrm{~mm}$ and $2.5 \mathrm{~mm}$. However, significantly thinner cement mantles were present in the proximal region in both groups (group 1, mean $1.578 \mathrm{~mm}$ (SD 0.140); group 2, mean $1.702 \mathrm{~mm}$ (SD 0.178)) compared with the middle region, where the thickest cement mantle was identified (group 1, mean 3.445 mm (SD 0.174); group 2, mean 2.82 mm (SD 0.170) (MannWhitney U test, $\mathrm{p}<0.05$ in both cases)).

New bone formation. The results for the area of new bone formation demonstrated comparable amounts with no significant difference when the mean was calculated for all regions between group $1\left(0.621 \mathrm{~mm}^{2}\right.$ (SD 0.032)) and group $2\left(0.610 \mathrm{~mm}^{2}\right.$ (SD 0.029) (Mann-Whitney U test, $\mathrm{p}>0.05))$. The mean for each region showed a trend in both groups, where the most bone was observed in the proximal (group 1, mean $0.859 \mathrm{~mm}^{2}$ (SD 0.055); group 2, mean $0.713 \mathrm{~mm}^{2}$ (SD 0.059) and distal (group 1, mean $0.703 \mathrm{~mm}^{2}$ (SD 0.048); group 2, mean $0.817 \mathrm{~mm}^{2}$ (SD 0.047 ), regions of the stem, with the least new bone formation in the middle (group 1, mean $0.424 \mathrm{~mm}^{2}$ (SD 0.049); group 2, mean $0.470 \mathrm{~mm}^{2}$ (SD 0.033)) and tip (group 1, mean $0.529 \mathrm{~mm}^{2}$ (SD 0.050); group 2, mean $0.440 \mathrm{~mm}^{2}$ (SD 0.055)) regions (Fig. 4b). No significant differences were identified between the two groups when the mean results of proximal, distal and tip regions were compared (Mann-Whitney U test, $\mathrm{p}>0.05$ )

ApaPore bone contact. The results demonstrated comparable amounts of bone contact within the graft, the mean result for all regions showing no significant difference between group $1(82.592 \% \quad(S D 1.05)$ and group 2 (81.534\% (SD 1.571) (Mann-Whitney U test, p > 0.05)). Similarly, the mean results demonstrated no significant differences between the groups when the percentage ApaPorebone contact was compared in a proximal to distal direction along the length of each stem (Fig. 4c).

Backscattered scanning electron microscopy. With this technique, bone growth was identified from the endosteal surface of the bone into and within the ApaPore granules in both groups (Fig. 5a). Haversian systems were observed adjacent to ApaPore which had been remodelled within mature lamellar bone. Additionally, in both groups bone was identified in contact with the outer surface of ApaPore, and also within its pores.

Histology. Histology showed complete reorganisation of the intramedullary canal. In both groups, the implant stem was surrounded by a layer of cement and this was surrounded by ApaPore granules, around and through which new bone had formed. The original cortex was evident.
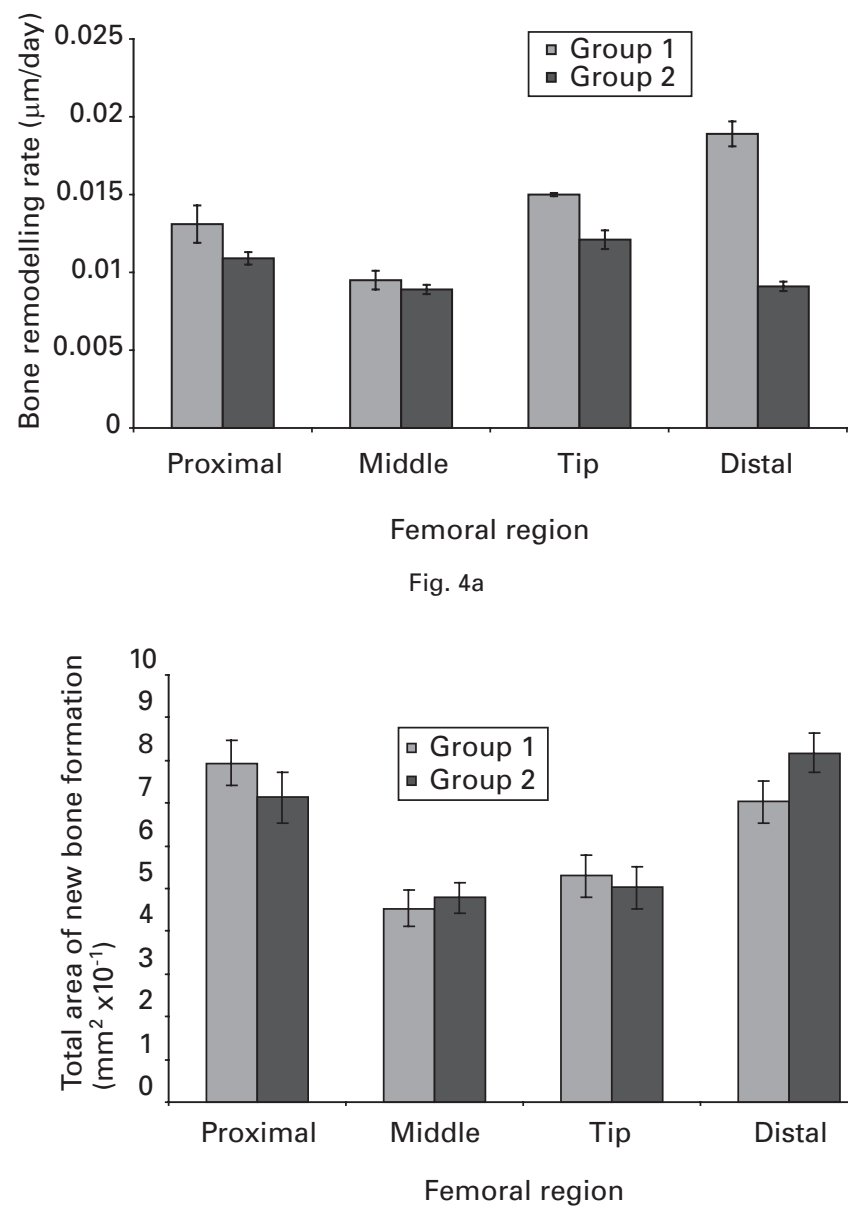

Fig. 4b

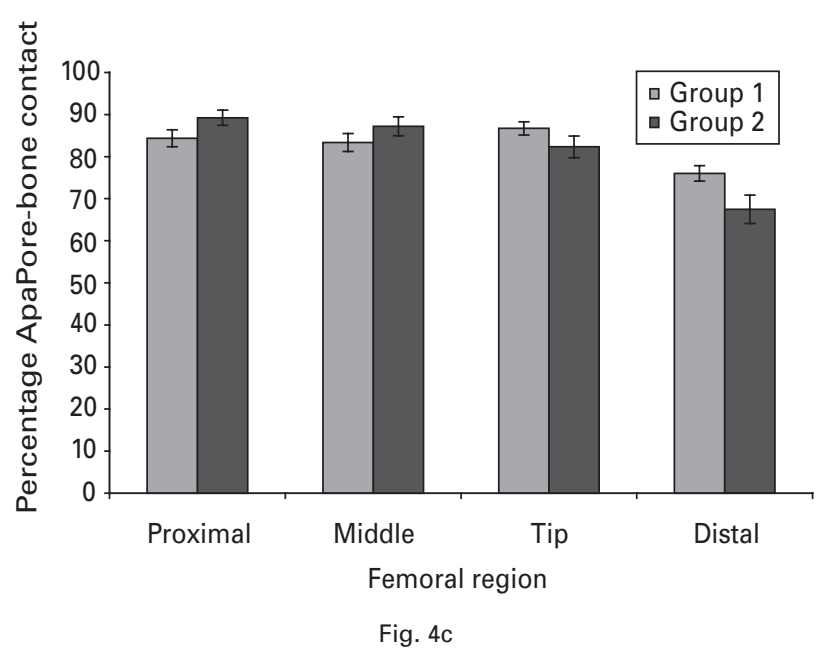

a) Histogram comparing the mean rates of bone formation in the two groups in each of the femoral regions. Error bars show the standard errors. b) A histogram comparing the mean total area of new bone formation in the two groups in each of the femoral regions. Error bars show the standard errors. The figures for the bone area are mean figures taken from a region of interest that measures $1.4 \mathrm{~mm}^{2}$. c) A histogram comparing the mean percentage ApaPore-bone contact in the two groups in each of the femoral regions. Error bars show the standard errors.

Histological and SEM analysis of specimens in both groups demonstrated abundant bone growth and direct contact in 


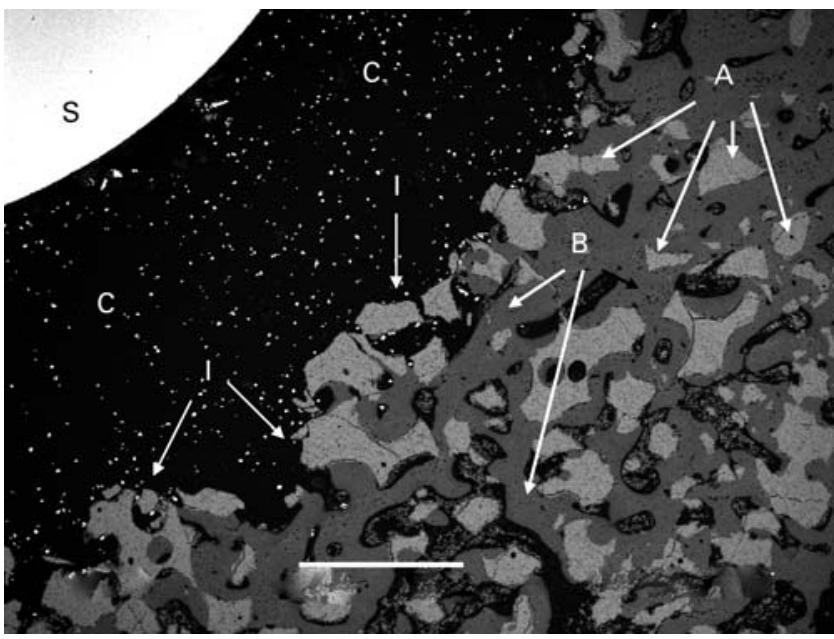

Fig. $5 a$

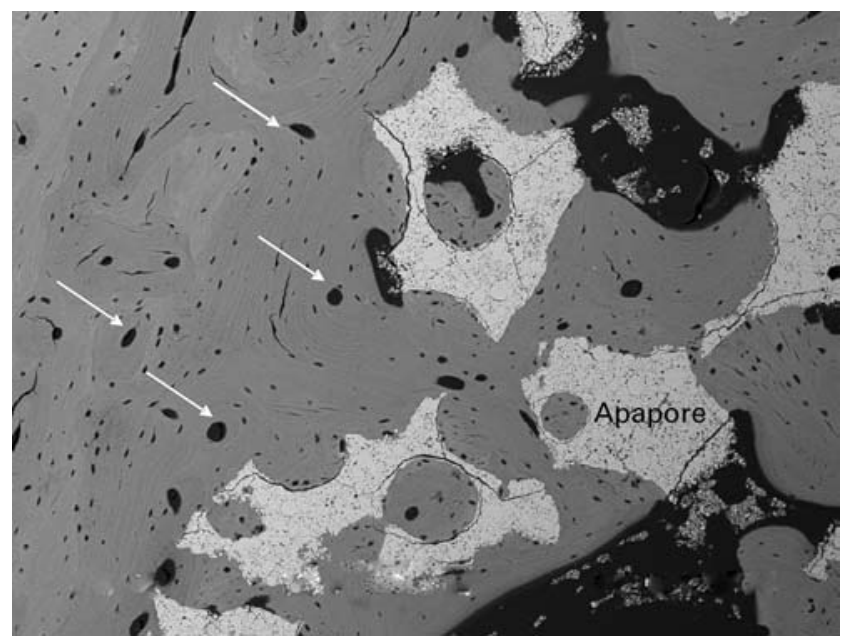

Fig. $5 b$

a) Backscattered electron micrograph showing the stem (S), cement (C), the graft with ApaPore particles (A) and new bone (B). Note the interdigitation of the cement with the ApaPore (I). Note also cracks in the ApaPore particles, which might have been associated with the impaction process. b) Backscattered electron micrograph showing pieces of ApaPore that have been remodelled within mature lamellar bone. Haversian systems (arrows) were observed adjacent to the ApaPore.

and around the ApaPore, forming a network of mature lamellar bone adjacent to the implant and within the medullary cavity (Fig. 5a). Broken particles of ApaPore were also evident, but were embedded within bone, and these were seen also in backscattered electron micrographs (Fig. $5 b)$. In many instances, bone lamellae within pores were centripedally organised, with bone forming preferentially on the walls of pores. Blood vessels were seen within the pores, and were often located centrally and surrounded by circumferentially orientated lamellar bone (Fig. 5b). The cement interface was interdigitated with both bone and ApaPore particles. At this interface, granules of ApaPore were identified in direct contact with the cement or were separated from it by a thin layer of bone (Fig. 6). ApaPore fragmentation was seen in both groups, where denselystained mononuclear, multinucleated and macrophage-like cells were present and contained submicrometre-sized particles of the bone substitute. Fragmentation occurred on ApaPore surfaces adjacent to fibrous tissue, whereas surfaces that were osseointegrated remained intact (Fig. 7).

\section{Discussion}

A limitation of this animal model is the inability to accurately recreate the tissue environment that remains after preparation for revision surgery of the hip. Other studies have documented the suitability of the ovine model for revision $\mathrm{THR}^{9,10}$ and reported that the architecture of the sheep femur replicates the human clinical situation, with a paucity of cancellous bone, a wide medullary cavity and a smooth endosteal surface. However, there are aspects of the tissue environment prior to revision THR in humans that are not represented in this model, including endosteal ero- sions, residual inflammatory tissue, and poor quality host bone.

We found no difference in the ground reaction force between the two groups post-operatively, indicating that all animals used their operated limb to the same extent. This provided quantitative evidence that the overall reduction in limb loading following surgery was not responsible for the difference in the results between groups. A reduction in the use of the limb may have implications for the amount of bone remodelling and new bone formation quantified within the graft mantle.

The thickness of the cement mantle was greatest in the middle region of the femoral component in both groups. No cement mantle was observed in the region distal to the tip of the stem. These results are supported by a recent study by Frei et al, ${ }^{11}$ who investigated the graft-cement-host bone interface after impaction allografting of a femoral component in human cadaver femora and showed significantly increased cement thickness in the middle region, with no cement immediately distal to the tip of the stem. They concluded that although cement-endosteal surface contact enhanced the strength of the allograft-cement composite-host bone interface, it was significantly weaker than a primary THR but considerably stronger than the cementless stem interface. Given these variations of morphology of the host bone interface along the femur in impaction grafting, one would expect the properties of the mechanical interface to vary. The cement-bone interface has been extensively studied in primary THR, but little is understood about its strength after impaction grafting. Frei et $\mathrm{al}^{11}$ suggested that the host bone interface may be enhanced by post-operative allograft remodelling, which 


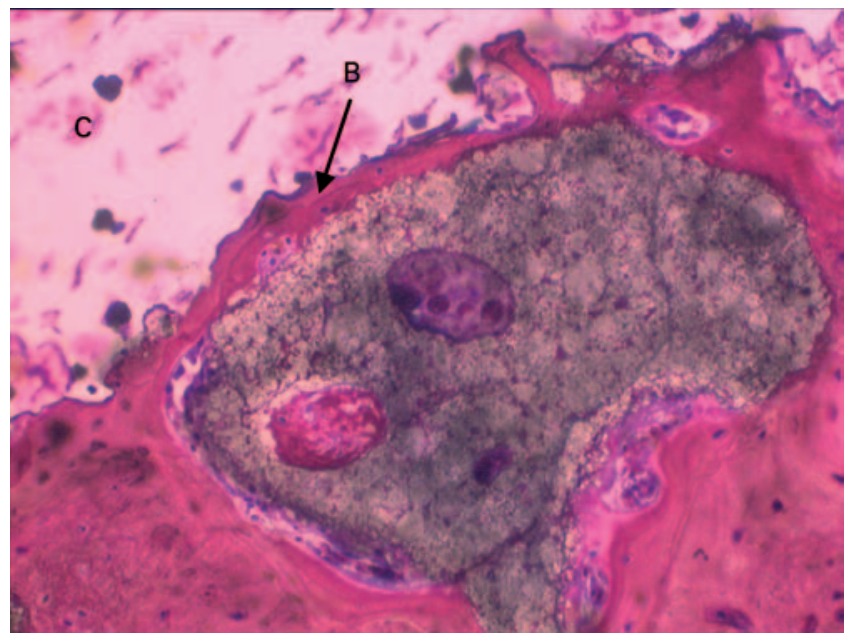

Fig. $6 a$

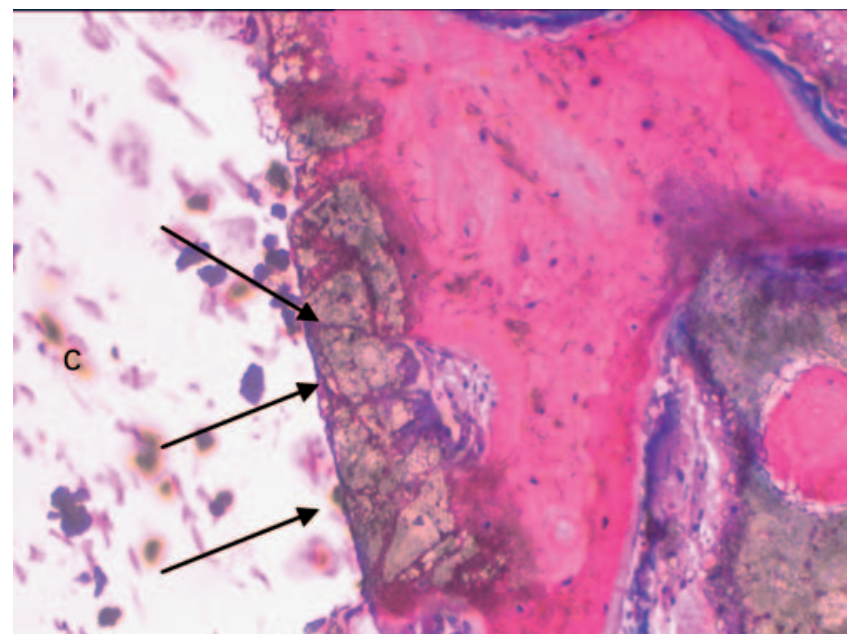

Fig. 6b

Photomicrographs showing a) the cement interface with ApaPore adjacent to the cement. The particles are separated from the cement (C) by a thin layer of bone (B), and b) the cement interface with particles of ApaPore (arrows) in direct contact with the cement (C).

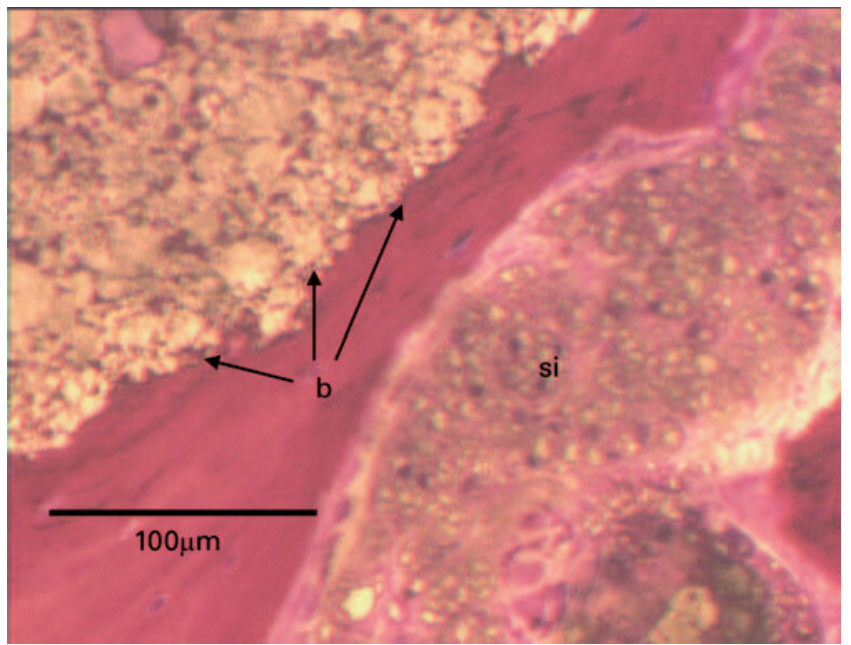

Fig. 7

Fragmentation of the ApaPore was only observed where soft-tissue interface (si) was present, and not where bone was in direct contact with the ApaPore (b).

may be the key to the success of the impaction allografting technique. Studies by Dai et $\mathrm{al}^{12}$ and Kwon et $\mathrm{al}^{13}$ have shown that inorganic bone particles and HA embedded in cement can be replaced by new bone and form a viable cement-bone interface. The shear strength of this interface was found to be significantly higher than that of cement alone. In our study, the results showed well-fixed implants in all cases, with extensive cement interdigitation within the ApaPore and allograft at the allograft-cement interface. Stem subsidence can occur through failure of the cement and the graft at interfaces between these materials and the stem or cortex. Munro et $\mathrm{al}^{14}$ performed an experiment in vitro to investigate whether increasing ratios of a synthetic porous HA material might also improve resistance to cumulative subsidence and cyclical movement on loading compared to $100 \%$ allograft in impaction grafting of the femur. Their results demonstrated that subsidence at the prosthesis-cement mantle and cyclical movement both significantly decreased with the addition of HA. Berzins et $\mathrm{al}^{15}$ showed no significant increase in the push-out strength of femoral components with increased cement penetration into the graft material. However, a study by Frei et $\mathrm{al}^{16}$ reported that cement penetration to the endosteal cortex would be beneficial for the clinical stability of the impaction allografting construct, but the excessive presence of cement would prevent extensive bone remodelling of the allograft and cortex. In addition, revascularisation of the endosteal cortex might be further delayed with excessive cement penetration. ${ }^{17}$

The administration of tetracycline bone markers allowed quantification of rates of bone turnover, and the results demonstrated significantly greater turnover in group 1 (50:50) than in group 2 (90:10). Previous studies support this finding. ${ }^{18,19}$ However, this study showed no significant difference in the amount of new bone formed following histological assessment of the two groups. The areas where fluoroescent markers had been incorporated within new bone were chosen randomly and at sites where it was possible for measurements to be made. In some areas there were very few sites where fluorescent markers could be identified. Therefore, these results represent the maximum bone turnover rates in each group and it is not possible to compare the bone turnover with the percentage of new bone formation. The rates of bone turnover as measured in 
this study only evaluated the potential of the ApaPore and allograft surfaces to form bone.

A study by Pratt et $\mathrm{al}^{6}$ compared impaction grafting of allograft alone with a 1:1 composite of allograft and a bone substitute comprised of $80 \%$ HA and $20 \%$ tricalcium phosphate in an ovine metaphyseal bone defect. The results demonstrated that larger quantities of new bone were measured within the group where allograft was impacted alone. This highlighted the improved integration and osseoconductive properties achievable with the use of allogeneic bone over synthetic materials. However, in our study we demonstrated equivalent amounts of bone formation in both groups, which may be a function of the limited osseoinductive properties of the allograft used which was subject to processing, gamma sterilisation and freezing prior to impaction within the femoral cavity. These procedures are known to destroy cells and bioactive factors present within the allograft, resulting in an osteoconductive scaffold on to which new bone will grow, but with weak or no osteoinductive capacity. ${ }^{20}$

To minimise variation in impaction, all specimens were prepared and manually impacted by the same person. However, some variation between the two groups could arise because of variable distribution of the particle sizes associated with greater amounts of bone graft in the 50:50 group and the reduced compressibility of HA compared to allograft. Deformation and intergranular motion can occur during compaction which can alter the quality of the impaction. Verdonschot et $\mathrm{al}^{21}$ reported that the size and porosity of the particles are important factors with less deformation exhibited in the biomaterial particles than in human bone graft. Compaction of the graft is essential for bone incorporation and stability of the implant, and will determine mechanical strain within the graft. ${ }^{22}$ Hydroxyapatite manufactured in particulate form can be harder than morcellised cancellous bone, and although this may affect the ease with which HA can be impacted, it will ultimately improve compaction and enhance stability of the implant.

Studies have shown that mechanical loading of the graft is important for maximum and rapid graft incorporation, with greater quantities of new bone ingrowth in loaded grafts than in non-loaded ones. ${ }^{23,24}$ An advantage of using bone substitutes such as ApaPore is that, provided the granules do not collapse, some porosity is present. Histological evidence from our study showed that, although there was fracture of some granules, there were also numerous pores through which blood vessels had penetrated. Increased microporosity of scaffolds has been shown to elicit rapid neovascularisation, which is essential for osseointegration. ${ }^{7}$

No significant differences in the percentage of ApaPorebone contact were identified when the two groups were compared. Histological and backscattered SEM analysis of specimens in all groups demonstrated a remodelled endosteal surface with abundant bone growth in direct contact within and around ApaPore granules, forming a network of mature lamellar bone adjacent to the implant and within the original medullary cavity. It is questionable whether the $10 \%$ allograft used in the group 2 (90:10) contributed to the remodelling of the graft.

As no immediate post-operative quantification of the presence of ApaPore within the femoral canals was performed it was not possible to state when the resorption of ApaPore began. However, following histological preparation of the specimens and observation using SEM, it was clear that the majority of ApaPore was still present, and in many cases completely surrounded by bone with no evidence of resorption at 24 weeks when the animals were killed. A previous in vitro study using a metallic tube and taper by Lawes and Buckland ${ }^{25}$ compared the use of allograft alone and in a 50:50 ApaPore:allograft mixture in impaction grafting in the hip, and demonstrated that the 50:50 group had 50\% greater mechanical stability, a 50\% decrease in subsidence and a significant reduction in the variability of the mechanical properties of the graft material.

The results of this study demonstrated a successful ovine model for hip impaction with well-fixed implants observed six months post-operatively, with no obvious signs of loosening or subsidence. The use of higher amounts of ApaPore in the allograft-bone substitute mixture revealed no significant differences between the groups when ground reaction force and bone growth were compared. In this animal model, the use of ApaPore was found to be a suitable alternative to replace allograft in impaction grafting. The authors conclude that further work is necessary to investigate the use of $100 \%$ ApaPore in impaction grafting, as $>90 \%$ ApaPore bone substitute would have significant advantages in terms of the sterility, immunogenicity, supply and consistency of the graft.

The authors thank Louisa Collins and Gillian Hughes for their assistance with this work, which was funded by ApaTech Ltd., Elstree, Hertfordshire, UK.

Although none of the authors has received or will receive benefits for personal or professional use from a commercial party related directly or indirectly to the subject of this article, benefits have been or will be received but will be directed solely to a research fund, foundation, educational institution, or other nonprofit organisation with which one or more of the authors are associated.

\section{References}

1. Dixon T, Shaw M, Ebrahim S, Dieppe P. Trends in hip and knee joint replacement: socioeconomic inequalities and projections of need. Ann Rheum Dis 2004;63:825-30.

2. Clohisy J, Calvert G, Tull F, McDonald D, Maloney W. Reasons for revision hip surgery: a retrospective review. Clin Orthop 2004;429:188-92.

3. Sporer SM, Paprosky WG. Femoral fixation in the face of considerable bone loss: the use of modular stems. Clin Orthop 2004;429:227-31.

4. Toms AD, Barker RL, Jones RS, Kuiper JH. Impaction bone-grafting in revision joint replacement surgery. J Bone Joint Surg [Am] 2004;86-A:2050-60.

5. Delloye C, Cornu 0, Druez V, Barbier 0. Bone allografts: what they can offer and what they cannot. J Bone Joint Surg [Br] 2007;89-B:574-9.

6. Pratt JN, Griffon DJ, Dunlop DG, Smith N, Howie CR. Impaction grafting with morsellised allograft and tricalcium phosphate-hydroxyapatite: incorporation within ovine metaphyseal bone defects. Biomaterials 2002;23:3309-17

7. Silva R, Camilli JA, Bertran CA, Moreira NH. The use of hydroxyapatite and autogenous cancellous bone grafts to repair bone defects in rats. Int J Oral Maxillofac Surg 2005;34:178-84. 
8. No authors listed. Home office. The animals (scientific procedures) act 1986. http:/ /www.archive.official-documents.co.uk/document/hoc/321/321-xa.htm (date last accessed 11 January 2008)

9. Goel VK, Drinker H, Panjabi MM, Strongwater A. Selection of an animal model for implant fixation studies: anatomical aspects. Yale J Biol Med 1982;55:113-22.

10. Eitel F, Klapp F, Jacobson W, Schweiberer L. Bone regeneration in animals and in man: a contribution to understanding the relative value of animal experiments to human pathophysiology. Arch Orthop Trauma Surg 1981;99:59-64.

11. Frei H, Mitchell P, Masri BA, Duncan C, Oxland TP. Mechanical characteristics of the bone-graft-cement interface after impaction allografting. J Orthop Res 2005;23:9-17.

12. Dai KR, Liu YK, Park JB, et al. Bone-particle-impregnated bone cement: an in vivo weight-bearing study. J Biomed Mater Res 1991;25:141-56.

13. Kwon SY, Kim YS, Woo YK, Kim SS, Park JB. Hydroxyapatite impregnated bone cement: in vitro and in vivo studies. Biomed Mater Eng 1997:7:129-40.

14. Munro NA, Downing MR, Meakin JR, Lee AJ, Ashcroft P. A hydroxyapatite graft substitute reduces subsidence in a femoral impaction grafting model. Clin Orthop 2007:455:246-52

15. Berzins A, Sumner DR, Wasielewski RC, Galante J0. Impacted particulate allograft for femoral revision total hip arthroplasty: in vitro mechanical stability and effects of cement pressurisation. J Arthroplasty 1996;11:500-6.

16. Frei H, O'Connell J, Masri BA, Duncan CP, Oxland TR. Biological and mechanical changes of the bone graft-cement interface after impaction allografting. J Orthop Res 2005;23:1271-9
17. de Waal Malefijt J, Sloof TJ, Huiskes R, de Laat EA, Barensz JO. Vascular changes following hip arthroplasty: the femur in goats studied with and without cementation. Acta Orthop Scand 1988:59:643-9.

18. Virolainen P, Heikkilä J, Yli-Urpo A, Vuorio E, Aro HT. Histomorphometric and molecular biologic comparison of bioactive glass granules and autogenous bone grafts in augmentation of bone defect healing. J Biomed Mater Res 1997;35:9-17.

19. Moore DC, Chapman MW, Manske D. The evaluation of a biphasic calcium phosphate ceramic for use in grafting long-bone diaphyseal defects. J Orthop Res 1987:5:356-65.

20. Dattani R, Blunn G. Revision of the femoral prosthesis with impaction allografting Acta Orthop Belg 2007;73:558-65.

21. Verdonschot N, Van Hal CT, Schreurs BW, et al. Time-dependent mechanical properties of HA/TCP particles in relation to morsellized bone grafts for use in impaction grafting. J Biomed Mater Res 2001;58:599-604.

22. Malkani AL, Voor MK, Fee KA, Bates CS. Femoral component revision using impacted morcellised cancellous bone graft: a biomechanical study of implant stability. J Bone Joint Surg [Br] 1996;78-B:973.

23. Van Der Donk S, Buma P, Verdonschot N, Schreurs BW. Effect of load on the early incorporation of impacted morsellised allografts. Biomaterials 2002;23:297303.

24. Wang JS, Tägil M, Aspenberg P. Load-bearing increases new bone formation in impacted and morsellised allografts. Clin Orthop 2000;378:274-81.

25. Lawes $\mathbf{P}$, Buckland T. Mechanical stability of simulated impaction grafts. Engineers and surgeons joined at the hip. IMechE June 2002, London, UK. 